\title{
On the relation of adaptation to field displacement during head movements to the constancy of visual direction'
}

HANS WALLACH AND LUCRETIA FLOOR ${ }^{2}$ SWARTHMORE COLLEGE

\begin{abstract}
When the normal constancy process on which the apparent immobility of the visual field during head movements is based was strengthened by the same method that produces adaptation to abnomal conditions in the constancy of visual direction, and when this training of the normal constancy process immediately preceded experimental adaptation, the effectiveness of the latter was diminished. This result applied not only to adaptation to horizontal field displacement and to vertical field displacement during turning of the head, but also to vertical field displacement during nodding of the head, a condition to which adaptation was here demonstrated for the first time.
\end{abstract}

When one considers instances of perceptual adaptation as learning problems, one of the most important issues may be the relation between the experimentally produced adaptation and the antecedents of the normal perceptual process which this adaptation modifies. The normal process is most likely a product of learning, an adaptation to the natural conditions of perceiving, as it were. Therefore, to understand experimental adaptation often requires seeing it in the context of the learning of the normal process. It is easy to establish evidence for this requirement. Where, after an adaptation has been produced, the effect dissipates spontaneously-that is, $S$ 's perceptual performance returns to normal during a mere time lapse, without an exposure apt to bring about adaptation to natural conditions--the the capacity for the normal process obviously has survived the experimental adaptation, and the normal process had only been temporarily superseded by it. To some degree such experimental adaptation resembles rote learning that takes place under strong proactive inhibition. The two processes differ in that the outcome of experimental adaptation and the normal perceptual process are mutually exclusive.

This being so, the existence of rapid partial adaptation that spontaneously dissipates raises a definite problem: How can experimental adaptation occur at all when, to become manifest, it temporarily supersedes rather than replaces the normal process that is the product of life-long practice? Two explanations may apply here. One is that the adaptation training temporarily overcomes the effect of life-long practice by the advantage of recency. The other assumes that the nature of the experimental adaptation period with its high concentration of the specific conditions that lead to adaptation is responsible. It contrasts with the infrequency with which corresponding conditions occur in ordinary life, where they lead to the building up of the normal process. Wallach and Karsh (1963) have obtained evidence that this latter explanation applies to the technique with which Wallach, Moore, and Davidson (1963) obtained rapid adaptation in stereoscopic vision to conditions that enhance the disparity with which objective depth is given. Wallach and Karsh strengthened the normal process immediately before adaptation training by exposing $S$ to conditions that corresponded to the experimental adaptation procedure, except that normal stimulation was employed instead of the adaptation condition that enhanced disparity. When this was done, subsequent adaptation training was found to be much less effective than when the pretraining in favor of the normal process was omitted. Thus, by training the normal process with the method of the adaptation training and by finding that this interferes with subsequent experimental adaptation, Wallach and Karsh made evident that the high effectiveness of the adaptation training can be ascribed to this method. It provided a concentration in time of the specific conditions apt to produce adaptation-either to enhanced disparity or to the natural conditions, as the case may be.

One of the purposes of the work to be here reported was to find out if adaptation in the constancy of visual direction (CVD) would show a corresponding effect of pretraining of the normal process. The term "constancy of visual direction" refers to the fact that the visual environment is normally perceived as stationary during head movements. A head movement will cause a displacement between the environment and the head. If such a relative displacement were caused by an objective displacement of the environment, that environment would be perceived in motion. Only if the displacement between environment and head is caused by a head movement does the environment appear stationary. Wallach and Kravitz (1965a) have found that the relative displacement of the environment must closely match the displacement of the head in order that the environment appear stationary. Even a small deviation from this match, as can be caused by an objective displacement of the environment during and dependent on the head movement, will cause the environment to be perceived in motion. That the stationary environment is perceived as stationary during a head movement is thus shown to be the result of a compensating process where the rate of head rotation is taken into account in the perception of motion or immobility of the environment-hence the use of the term "constancy." It is most likely that this compensating process is the result of an adaptation to the natural conditions. This normal process, in turn, can be altered by experimental adaptation. ${ }^{3}$ Wallach and Kravitz (1965b) and Wallach and Frey (1969) have demonstrated that partial adaptation of this kind can be obtained very rapidly, and the latter have demonstrated spontaneous dissipation of the effect of experimental adaptation. In these experiments, the specific conditions leading to adaptation consist, of course, in head movements that displace the eyes relative to the visual environment. If the environment remains objectively stationary during head movements, the normal process is strengthened, whereas actual displacements of the field content during and dependent on the head movements in some constant proportion leads to experimental adaptation. The natural conditions under which the normal process produces a stationary perceived environment consist in displacements of the environment relative to the head brought about by the combination of a head movement with a stationary environment. If, for instance, the head is turned to the right, the stationary environment is displaced relative to the head to the left in an amount that matches the head rotation. Any sizable deviation from the matching displacements of the natural conditions causes experimental 
adaptation. Such a deviation is, of course, brought about by an objective displacement of the environment in the direction with or against the head movement. ${ }^{4}$

Our procedure corresponded to that of Wallach and Karsh: Our Ss underwent the same experimental adaptation on two occasions. On one of them it was preceded by adaptation to the natural conditions, consisting of ceaseless turning movements of the head while a stationary environment was viewed. By comparing the effectiveness of the immediately following experimental adaptation training with that of the experimental adaptation obtained on the other (control) occasion, we could demonstrate a possible detrimental effect that the preadaptation training of the normal process has on the experimental adaptation. To get more information on the manner in which this pretraining affects the developing experimental adaptation, the training of the latter was twice briefly interrupted for testing. This was made possible by using the one-trial test first employed by Wallach and Frey (1969).

Up to the present, adaptation in the constancy of visual direction had been produced only for head-turning movements that consisted of rotations in the horizontal plane. In Experiment 2 we employed nodding, in part a head rotation in a vertical plane, that is, about a horizontal axis that extends from left to right. We repeated Experiment 1 with the same Ss, employing nodding of the head and replacing for experimental adaptation objective horizontal displacements of the environment by vertical ones.

Recently, Wallach, Frey, and Romney (1969) demonstrated partial adaptation to vertical displacements of the environment that occur during and dependent on head turning, that is, rotation in the horizontal plane. The usual experimental adaptation is to objective field displacement whose direction is in the plane of the head rotation; vertical field displacement is perpendicular to the plane of the head rotation. The authors called this adaptation "unrelated to the constancy of visual direction," because such objective displacements do not enhance or diminish the normal displacement of the field, relative to the head, that a head movement produces under natural conditions. After we had found in Experiments 1 and 2 that pretraining of the normal process has a detrimental effect on experimental adaptation, we questioned whether this would also be true in the case of experimental adaptation unrelated to the constancy of visual direction. The answer was obtained by Experiment 3, which was identical with Experiment 1 except that experimental adaptation was to vertical field displacement while the head turned in the horizontal plane.

\section{METHOD}

The rapid-adaptation procedure of Wallach and Frey (1969) was employed in all three experiments and the slant-estimation technique developed by these authors was used in all tests. The original CVD test apparatus described by Wallach and Kravitz (1968) and equipped with two mirrors for the work of Wallach and Frey ${ }^{5}$ served in Experiments 1 and 3, while a new device suited to head nodding, which will be described below, was used in Experiment 2.

\section{Experiment 1}

Here, the slant-estimation test measures the apparent horizontal displacement of a stationary luminous disk in the dark during head movements; this is a manifestation of experimental adaptation to a horizontal field displacement during head turning. By combining this apparent horizontal displacement with an actual vertical displacement, it is transformed into an oblique motion path, which is the kinematic resultant of the apparent horizontal and the actual vertical displacement. With the vertical displacement component at a fixed rate (of $40 \%$ of the head rotation in all tests), the slant angle of the apparently oblique motion path measures the rate of the apparent horizontal displacement component. $\mathbf{S}$ reproduced this slant angle by setting a rod that could be turned in a frontal-parallel plane to the same apparent slant. As had been done by Wallach and Frey, our Ss were given preliminary tests in slant estimation by presenting them with a series of 16 objectively oblique motion paths of various slant angles. This gave our Ss practice with this type of test and made it possible to eliminate those with a poor performance. The procedure described by Wallach and Frey under PRELIMINARY EXPERIMENTS (2) was followed. A few Ss whose slant-estimation scores (average of two settings) showed more than three reversals were eliminated.

The experiment consisted of two sessions. Each began with the preliminary tests, followed by a preadaptation test in which $S$ gave a slant estimate for an objectively vertical motion path. He observed, for three full head movements, a dimly luminous disk in the dark move upwards when he turned his head to the right and downwards when the head movement was to the left, and then made his rod setting. The session called "experimental" continued with training of the normal constancy process: $S$ made 200 full head movements while fixating the disk which now was at rest, representing a stationary environment. This pretraining was immediately followed by three periods of experimental adaptation, each ending in a postadaptation test of slant estimation. The latter were identical with the preadaptation test except that $S$ was allowed only two full head movements. ${ }^{6}$ For the purpose of experimental adaptation, the disk was being displaced horizontally at a rate of $40 \%$ of the head rotation. (In all three of our experiments, the same displacement ratio of $40 \%$ was employed for experimental adaptation.) The three periods of experimental adaptation lasted for 50,100 , and 200 full head movements, with each immediately following the postadaptation test for the preceding period. The session ended with a measurement of spontaneous dissipation: $\mathrm{S}$ sat quietly with eyes closed for $5 \mathrm{~min}$ and then made a last slant estimate.

The control session was identical with the experimental session just described, except that the pretraining to a stationary disk was omitted. The two sessions were separated by a week. Half of our 16 Ss first served in an experimental session and then in a control session, while for the other half the sequence was reversed. There are two kinds of horizontal field displacements that depend on head turning; the displacement may be in the direction with the head movement or against it. For half of our Ss experimental adaptation was to a disk that shifted in the direction with the head rotation, and for the other half its displacement was in the opposite direction.

\section{Experiment 2}

Here the head movement was a nodding, and experimental adaptation was to vertical disk displacement in the direction of either with or against the head rotation implicit in the nodding movement. In the test, the actual displacement of the disk was horizontal and a deviation of its apparent motion path from horizontal measured the adaptation effect.

A new transmission was built specifically meeting these requirements. Since all displacement rates amounted to the same $40 \%$, a simple device with a fixed ratio could be used. The input shaft, which could be attached to a headgear and was to be aligned with the horizontal rotation axis of the nodding movement, ended in the larger of a pair of gears that reduced its rotation rate five to one. The smaller gear turned the primary output shaft, which ended in a mirror that reflected a beam of projector light onto a screen $2.5 \mathrm{~m}$ in front of $S$ where it formed the disk. This mirror thus turned about a horizontal axis when 
S's head nodding turned the input shaft and thus displaced the disk upward when the head turned down and vice versa at the desired rate of $40 \%$. (The mirror turned at a rate of $20 \%$ of the head rotation, but the reflected beam, of course, changed its direction by twice this amount.) There were two further output shafts. One, horizontal as the primary shaft, was connected to it by a pair of gears of equal size, causing its rotation direction to be reversed. Therefore, while the primary shaft turned the mirror in the direction opposite to the head movement, attaching the mirror to this secondary shaft caused the disk to become displaced in the direction with the head movement. The other secondary shaft was vertical and attached to the primary shaft by a pair of right-angle gears. A second mirror, when ridigly connected with this shaft, would displace a projector beam it reflected in the horizontal plane. The attachment of this mirror was so arranged that it could be put in a fixed position, which placed a stationary disk in the center of the screen, or it could be made to turn with the shaft. This device thus provided all the conditions of our experiment: A mirror attached to one or the other horizontal shafts would cause the disk displacement in the direction with or against the head movement employed in the two conditions of experimental adaptation, and the other mirror would provide a stationary disk for the pretraining when it was fixed in one position and the horizontal disk displacement for the slant-estimation test when it was made to tum with the vertical shaft. There were two projectors located to S's side whose beams were directed to the turning mirrors by a large stationary mirror obliquely in front of $\mathrm{S}$. One projector's beam was reflected by the vertically displacing mirror when it was attached to either one of the horizontal shafts. The other projector's beam was aimed at the mirror located on the vertical shaft and would provide the stationary or the horizontally displacing disk. Changing from one of the adaptation conditions to a test or to the pretraining condition required switching current from one projector to the other, whereas to change the direction of the vertical disk displacement, which was done only between experiments, necessitated shifting a mirror from one horizontal shaft to the other.

The headgear used to transmit the rotational component of the nodding to the input shaft of the transmission consisted of a stripped-down welder's headgear to one side of which an adjustable mounting for a rod was attached which transferred the rotational component to the input shaft. This mounting consisted essentially of two slotted sliding bars, a vertical and a horizontal one. The vertical one could be raised or lowered along a vertical bar permanently attached to the headgear. A key and recess arrangement between this pair of bars permitted only vertical adjustment, and the movable bar could be fixed by tightening a screw, giving the pair the desired length. Cut into the free lower end of the vertical sliding bar was another key whose guiding edges were horizontal. It fitted the recess in the second sliding bar, which thus formed a right angle with the first one and could be adjusted frontward or backward relative to the headgear. The rod that served to transfer the head rotation to the transmission was perpendicularly attached to the rear end of he horizontal bar. When the headgear was that the rod coincided with the axis of the head rotation, which was located in the upper neck. ${ }^{7}$ S's chair could be raised or lowered to the point where the rod could be attached to the transmission that was in permanent position on the side of the chair. All surfaces in S's visual field reflecting stray light from the projectors were masked with black material.

Except that the preliminary slant-estimation tests were omitted, the procedure in Experiment 2 was analogous to that of Experiment 1. The same $16 \mathrm{Ss}$ were employed and the groups who had been adapted to horizontal disk displacement in the direction with the head movement or against it were given the analogous vertical displacements.

\section{Experiment 3}

The apparatus used in Experiment 1, which fits head turning, was employed. Now the vertically displacing mirror was used for experimental adaptation, while the other caused the horizontal motion path for the test. In every other respect, this experiment was the same as Experiment 1, except that the preliminary slant-estimation tests were again omitted and that there was only one combination of head movement and disk displacement: The disk moved up when $S$ turned his head to the right and down with left tuming. worn, the two sliding bars could be set so

Because some were no longer available, only 5 of the original $16 \mathrm{Ss}$ were among the 12 who participated in Experiment 3.

\section{RESULTS}

The mean adaptation effects of all three experiments are presented in Table 1 . Adaptation scores were obtained by deducting from each postadaptation slant estimate the preadaptation slant estimate obtained in the same session. These estimates were the slant angles of the rod settings in degrees. An analysis of variance of the results of Experiments 1 and 2 shows a significant difference between the experimental and control conditions $(\mathrm{F}=9.25, \mathrm{p}=.01)$ but no significant difference between the two experiments $(F=1.77)$ or for the order in which Ss served in the experimental and control conditions $(F=2.71)$. The differences between the mean adaptation scores obtained in the experimental and control conditions were quite large, particularly after the first 50 head movements, where they were significant in all experiments (in Experiment 1, $p=.025$, and in the other two experiments, $p=.01$ ). Another clear result was the growth of experimental adaptation with increase in training; the difference between mean adaptation scores after 50 head movements and after an additional 100 head movements was significant in five out of six conditions. It also seems clear that the growth in adaptation slows as a higher level of adaptation is achieved, a result comparable to that obtained by Wallach, Moore, and Davidson (1963) for adaptation in stereoscopic depth perception. The retest after a lapse of 5 min showed a significant decline in the adaptation effect in Experiments 1 and 2. This result confurmed an earlier finding by Wallach and Frey pertaining to head turning and showed that spontaneous dissipation of experimental adaptation occurs also when the adaptation is to field displacement during nodding. The decline that occurred in Experiment 3 was much smaller, but the difference between these losses in Experiments 1 and 3 was not significant.

There was no significant difference

Table 1

Mean Adaptation Scores Computed from Slant Angle Setting

\begin{tabular}{|c|c|c|c|c|c|c|c|c|}
\hline \multirow{2}{*}{$\begin{array}{l}\text { Experi- } \\
\text { ment }\end{array}$} & \multirow{2}{*}{ Condition } & \multicolumn{5}{|c|}{$\begin{array}{c}\text { After Different Numbers } \\
\text { of Head Movements }\end{array}$} & \multirow{2}{*}{$\begin{array}{l}\text { After } \\
5 \text { Min } \\
\text { Lapse }\end{array}$} & \multirow{2}{*}{$\begin{array}{c}\text { Significance of } \\
\text { Difference Between } \\
\text { Means from Last and } \\
\text { Preceding Column }\end{array}$} \\
\hline & & 50 & plus & 100 & plus & 200 & & \\
\hline 1 & $\begin{array}{l}\text { Control } \\
\text { Experimental }\end{array}$ & $\begin{array}{l}4.3 \\
1.7\end{array}$ & & $\begin{array}{l}5.6 \\
3.9\end{array}$ & & $\begin{array}{l}6.8 \\
5.8\end{array}$ & $\begin{array}{l}3.6 \\
3.9\end{array}$ & $p=.02$ \\
\hline 2 & $\begin{array}{l}\text { Control } \\
\text { Experimental }\end{array}$ & $\begin{array}{l}3.4 \\
0.8\end{array}$ & & $\begin{array}{l}6.0 \\
3.2\end{array}$ & & $\begin{array}{l}6.8 \\
5.1\end{array}$ & $\begin{array}{l}4.0 \\
3.0\end{array}$ & $p<.01$ \\
\hline 3 & $\begin{array}{l}\text { Control } \\
\text { Experimental }\end{array}$ & $\begin{array}{l}5.4 \\
0.7\end{array}$ & & $\begin{array}{l}7.6 \\
4.8\end{array}$ & & $\begin{array}{l}8.1 \\
5.4\end{array}$ & $\begin{array}{l}6.6 \\
3.9\end{array}$ & $p>.2$ \\
\hline
\end{tabular}


between the mean adaptation scores of the groups of eight Ss each whose adaptation was to disk displacement in the direction with the head rotation or against it. Wallach and Frey (1969), who also used rapid training and slant estimation tests, had found significantly higher adaptation effects when training was in the direction with the head turning than when it was in the opposite direction, and Wallach, Frey, and Romney (1969) had obtained a corresponding result with 1-h-long adaptation periods and a test that involved measurements of no-motion ranges. (In the present experiment, the mean adaptation scores from the control sessions were, in fact, higher-but not significantly-for those eight Ss who adapted to disk displacement against the head rotation than for those who had the disk displaced in the opposite direction, and this was true for head turning as well as for head nodding.) This loss of significance results from the use of different groups of only eight Ss in the two adaptation conditions (with the same groups assigned to corresponding conditions in Experiments 1 and 2), whereas in the previous experiments the same $S s$ were used. It is a manifestation of the large differences in the amount of experimental adaptation measured in the case of different individuals.

\section{DISCUSSION}

Training the normal constancy process just prior to experimental adaptation training was found to diminish the adaptation effect. This result is analogous to one obtained by Wallach and Karsh (1963) and suggests that the capability of experimental adaptation to supersede temporarily the normal constancy process is, at least in part, due to the method used in the adaptation training. For when this method, which provided concentration in time of the specific conditions that produce adaptation, was used to strengthen the normal constancy process, the effect of an immediately succeeding adaptation training was diminished.

Our result is also an indication of an interference on the part of the normal constancy process with experimental adaptation. The notion of such an interference was originally derived from the fact earlier established and confirmed in the present experiment that, after training has ended, a mere lapse of time diminishes the effect of experimental adaptation.

Wallach, Frey, and Romney (1969) have called adaptation to vertical field displacement during and dependent on head rotation in the horizontal plane as occurs in Experiment 3 "unrelated to the constancy of visual direction." This terminology derives from the view that the constancy of visual direction is the result of an adaptation to the displacements relative to the head that a stationary field undergoes during head movements. This relative displacement of an objectively stationary field occurs, of course, in the plane of the head rotation, i.e., horizontally in the case of head tuming. Objective field displacements in the direction with or against the head rotation diminish or augment this relative displacement when they, too, are considered as displacements relative to the head. The vertical field displacement of Experiment 3, on the other hand, when it is considered a displacement relative to the head causes that displacement to become oblique in relation to the plane of the head rotation. With the vertical disk displacement amounting to $40 \%$ of the head rotation, the disk's path formed an angle of nearly $22 \mathrm{deg}$ with the horizontal plane. The difference between constancy-related adaptation and constancy-unrelated adaptation then amounts to this: The former is to diminished or augmented field displacement, while the latter is to field displacement in a direction different from that which occurs under normal conditions. The question arose as to whether or not this difference entailed essentially different interference between the normal process and experimental adaptation. We found that, where the effect of pretraining is concerned, it did not.

\section{REFERENCES}

WALLACH, H., \& FREY, K. J. Adaptation in the constancy of visual direction measured by a one-trial method. Perception \& Psychophysics, 1969, 5, 249-252.

WALLACH, H., FREY, K. J., \& ROMNEY, G. Adaptation to field displacement during head movement unrelated to the constancy of visual direction. Perception \& Psychophysics, 1969, 5, 253-256.

WALLACH, H., \& KARSH, E. G. Why the modification of stereoscopic depth perception is so rapid. American Journal of Psychology, $1963,76,413-420$.

WALLACH, H., \& KRAVITZ, J. H. The measurement of the constancy of visual direction and of its adaptation. Psychonomic Science, 1965a, 2, 217-218.

WALLACH, H., \& KRAVITZ, J. H. Rapid adaptation in the constancy of visual direction with active and passive rotation. Psychonomic Science, 1965b, 3, 165-166.

WALLACH, H., \& KRAVITZ, J. H. Adaptation in the constancy of visual direction tested by measuring the constancy of auditory direction. Perception \& Psychophysics, 1968, 4, 299-303.

WALLACH, H., MOORE, M. E., \& DAVIDSON, L. Modification of stereoscopic depth-perception. American Journal of Psychology, 1963, 76, 191-204.

\section{NOTES}

1. The work was supported by Grant 11089 from the National Institutes of Health to Swarthmore College, Hans Wallach principal investigator.

2. Address: Department of Psychology, Swarthmore College, Swarthmore, Pennsylvania 19081.

3. A general introduction to adaptation in the constancy of visual direction may be found in Wallach and Kravitz (1968).

4. For a more detailed explication of the relation between experimental adaptation and the normal process, see Wallach and Frey (1969), p. 249.

5. An improvement was made for the work to be reported: To provide for a more rapid change from horizontal to vertical target displacement, the vertically displacing mirror was connected to the input shaft of the variable transmission by an electric clutch and break. The horizontally displacing mirror could be arrested quickly by setting the variable transmission to zero.

6. The vertical displacement of the disk presented during these head movements is equivalent to a stationary disk. Thus, the test amounts to a brief training of the normal constancy process, which would tend to diminish the experimental adaptation already achieved. Employing the smallest feasible number of head movements was therefore desirable.

7. We thank Mr. Otto Hebel for designing and constructing the transmission and the headgear mounting.

(Accepted for publication October 27, 1969.) 